\title{
The Phase A study of the ESA M4 mission candidate ARIEL
}

\author{
Ludovic Puig $^{1} \cdot$ Göran Pilbratt $^{1}$ • Astrid Heske ${ }^{1} \cdot$ Isabel Escudero ${ }^{1}$. \\ Pierre-Elie Crouzet ${ }^{1} \cdot$ Bram de Vogeleer $^{2} \cdot$ Kate Symonds $^{2} \cdot$ Ralf Kohley $^{3}$. \\ Pierre Drossart ${ }^{4}$. Paul Eccleston ${ }^{5} \cdot$ Paul Hartogh $^{6}$. Jeremy Leconte ${ }^{7}$. \\ Giusi Micela ${ }^{8} \cdot$ Marc Ollivier $^{9,4} \cdot$ Giovanna Tinetti $^{10} \cdot$ Diego Turrini $^{11,12}$. \\ Bart Vandenbussche ${ }^{13}$. Paulina Wolkenberg ${ }^{11,14}$
}

Received: 22 January 2018 / Accepted: 31 August 2018/Published online: 13 September 2018

(C) The Author(s) 2018

\begin{abstract}
ARIEL, the Atmospheric Remote sensing Infrared Exoplanet Large survey, is one of the three M-class mission candidates competing for the M4 launch slot within the Cosmic Vision science programme of the European Space Agency (ESA). As such, ARIEL has been the subject of a Phase A study that involved European industry, research institutes and universities from ESA member states. This study is now completed and the M4 down-selection is expected to be concluded in November 2017. ARIEL is a concept for a dedicated mission to measure the chemical composition and structure of hundreds of exoplanet atmospheres using the technique of transit spectroscopy. ARIEL targets extend from gas giants (Jupiter or Neptune-like) to super-Earths in the very hot to warm zones of F to M-type host stars, opening up the way to large-scale, comparative planetology that would place our own Solar System in the context of other planetary systems in the Milky Way. A technical and programmatic review of the ARIEL mission was performed between February and May 2017, with the objective of assessing the readiness of the mission to progress to the Phase B1 study. No critical issues were identified and the mission was deemed technically feasible within the M4 programmatic boundary conditions. In this paper we give an overview of the final mission concept for ARIEL as of the end of the Phase A study, from scientific, technical and operational perspectives.
\end{abstract}

Keywords ARIEL · Cosmic vision · M4 · ESA · Phase a study $\cdot$ Exoplanets · Transmission and eclipse spectroscopy

Ludovic Puig

ludovic.puig@esa.int

Extended author information available on the last page of the article 


\section{Introduction}

ARIEL is a M4 mission candidate within the Cosmic Vision science programme of ESA. ARIEL was selected in June 2015 to enter an assessment phase (Phase 0/A), and is competing against two other missions (THOR and XIPE) for the M4 launch slot in 2026. The ARIEL mission is the successor of the ESA M3 mission candidate EChO [1]. The science objectives and mission concept have evolved to fit the new M4 programmatic constraints. The main changes are the use of a new European launcher (Ariane 6 instead of Soyuz), a new launch date (2026 instead of 2024) and a slightly reduced cost at completion to ESA. Other programmatic constraints specific to the M-class missions still apply (Technology Readiness Levels, payload and S/C development time).

The Phase 0 study of ARIEL involved the preliminary definition of the science and mission requirements, and an ESA internal Concurrent Design Facility (CDF) study conducted in June/July 2015. The results of this CDF study are presented in [2].

Following this Phase 0, the Phase A study was kicked-off in early 2016, with two parallel competitive industrial studies as well as a payload study. This study culminated in the Mission Selection Review (MSR) in February-May 2017, with the objective of assessing the technical and programmatic feasibility of all three M4 mission candidates. With a successful conclusion to the review, all three candidate missions are now undergoing an independent scientific evaluation. This process is expected to be concluded in November 2017 with the selection of the M4 mission by the ESA Science Program Committee (SPC).

After November 2017, the selected M4 mission will enter a Phase B1 study that is expected to conclude in mid-2019 with a Mission Adoption Review (MAR).

In this paper we present a summary of the key aspects of the ARIEL phase A study. In section 2 we give a brief overview of the science, science requirements and targets of the mission; in section 3 we provide an overview of the mission requirements, followed by a description of the mission profile (including operations) in section 4; in sections 5 and 6 we describe the spacecraft design and the assembly/integration/verification/ development plan respectively, with a summary of the way in which the Community would be able to access ARIEL given in section 7. In section 8 we present the findings and recommendations of the MSR.

\section{Science requirements and targets}

\subsection{Background and key science objectives}

ARIEL is a survey-type mission concept dedicated to the study of exoplanets through spectral characterisation of their atmospheres. Currently it is predicted that most stars in our Galaxy contain one or more planets, thus in our galaxy alone $\sim 10^{11}$ or more exoplanets likely exist. Today a few thousand exoplanets have been actually identified, displaying huge ranges of masses, sizes and orbits: from rocky Earth-like planets to large gas giants grazing the surface of their host star. However, the essential nature of these exoplanets remains largely unknown: there is no known, discernible pattern linking the presence, size, or orbital parameters of a planet to the nature of its parent star. We have little idea how the chemistry of a planet is linked to its formation 
environment, or whether the type of host star drives the physics and chemistry of a planet's birth, and subsequent evolution.

The next step in exoplanet science is to study the planets themselves, both individually and importantly statistically, with a view to attempt to understand what they are, and why they are - and how they have become - what they are. ARIEL is designed as a dedicated survey mission capable of observing a large and well-defined planet sample of transiting exoplanets within its baseline 4-year mission lifetime. The top-level science objectives are to address the fundamental questions:

- What are exoplanets made of?

- How do planets and planetary systems form?

- How do planets and their atmospheres evolve over time?

This will be done through the direct measurement of the chemical composition and thermal properties of a large population of exoplanets. The diversity in compositions is expected to be linked to different formation and evolution scenarios. ARIEL will therefore observe spectroscopically approximately 1000 transiting planets of different sizes, masses, densities, equilibrium temperatures, and orbital properties around a variety of stellar types to establish what these planets are made of.

By employing time differential techniques of transit, eclipse, and phase-curve spectroscopy, whereby the signal from a star and planet system is differentiated using knowledge of the planetary ephemerides, it is possible to measure atmospheric signals from the planet at levels of 10-100 ppm (ppm) relative to the star. Particularly bright targets will also allow more sophisticated techniques, such as eclipse mapping, to be employed promising an even deeper insight into the nature of planetary atmospheres. Photometric stability rather than angular resolution is key, and is in fact the most stringent requirement of the ARIEL design driving many engineering design and operational aspects of the mission. For the brightest targets it would be possible to obtain high quality spectra in a single visit; for fainter targets the necessary signal-tonoise would be built up through repeated visits over the mission lifetime.

The instantaneous spectral coverage of the ARIEL design is unique in its breadth, spanning the 1.25 to $7.8 \mu \mathrm{m}$ range with three contiguous spectrometer bands, and in addition three narrow-band photometry channels in the optical. The spectral range is primarily aimed for the study of warm and hot exoplanets with temperatures ranging from several hundred to over a few thousand Kelvin, taking advantage of their well-mixed atmospheres which should show minimal condensation and sequestration of high- $Z$ materials and thus better reveal their bulk elemental composition (especially $\mathrm{C}, \mathrm{O}, \mathrm{N}, \mathrm{S}$, $\mathrm{Si}$ ). The wavelength range covers all the expected major atmospheric gases from e.g. $\mathrm{H}_{2} \mathrm{O}$, $\mathrm{CO}_{2}, \mathrm{CH}_{4} \mathrm{NH}_{3}, \mathrm{HCN}, \mathrm{H}_{2} \mathrm{~S}$ through to the more exotic metallic compounds, such as TiO, $\mathrm{VO}$, and condensed species. Furthermore, the broad instantaneous spectral coverage, importantly including the visible bands, provides an essential means by which to monitor and subsequently correct for the effects of activity of the host star, which could otherwise introduce significant uncertainty into the final exoplanet spectrum and its interpretation.

Progress with the science questions requires a large, unbiased spectroscopic survey of exoplanets. The ARIEL mission would allow scientists to study exoplanets both as a population and as individuals. It is designed to target super-Earths, Neptune-like, and Jupiter-like planets, in the warm to very hot zones (planet temperatures of $\sim 500$ 
$3000 \mathrm{~K}$ ) of F to M-type host stars. The spectroscopic information on the atmospheres of the large, select sample of exoplanets that ARIEL would provide would allow the compositions, temperature profiles, size, diversity and variability to be determined to levels of accuracy and precision never previously attempted.

A much more detailed description of the science case for ARIEL as well as the key science objectives of the mission can be found in chapter 2 of the ESA ARIEL Assessment Study Report [3], as well as in [4], and references therein.

\subsection{Scientific requirements and observational strategy}

The high-level science objectives of ARIEL flow down to a number of science requirements, both on the breadth and depth of the survey of exoplanets to be undertaken by ARIEL, as well as on the science requirements for the mission itself, see also chapter 3 in [3].

\subsubsection{Observational strategy}

The ARIEL science objectives call for atmospheric spectra of a large and diverse sample of known exoplanets covering a wide range of masses, densities, equilibrium temperatures, orbital properties and host-star taken at various spectral resolving powers, wavelength intervals and signal-to-noise ratios (SNRs). This can be achieved through the execution of observation of a large enough well chosen sample of $>500$ exoplanet targets, with a goal to observe $\sim 1000$ exoplanet targets.

The ARIEL observational strategy is to divide the overall survey into three tiers Reconnaissance survey, Deep survey, and Benchmark planets. The Reconnaissance survey mode will allow rapid, broad characterisation of planets so that decisions can be made about priorities for future observations. For the majority of the targets observed by ARIEL, the necessary performance can be reached in just 1 or 2 transits/eclipses. The Deep survey will perform spectroscopy observations of a sub-sample, for the majority of the targets observed the required performance can be reached with 1 to 10 transits/eclipses. The Benchmark planet observations will focus in particular on the study of the variability through time of the exoplanet atmospheres (weather). Repetition through time of the same observations will cast light on the temporal variability of the exo-atmospheres due to variations in the cloud coverage or patterns in the global circulation.

An example summary of the tiers, observational strategies, and associated science cases for each tier is provided in Table 1. A detailed breakdown of the observing time between the different tiers has not been fixed during the study, however, in order to achieve the required diversity there is a formal requirement to observe at least 500 planets. Simulations indicate - depending on the details on how the survey is optimised - that approximately 1000 targets can be observed, with the breakdown in numbers and fractions of observing time per tier shown.

These simulations and optimisations will continue until - and during - the actual flight of ARIEL. The target list definition process will involve the general astronomical community and the ESA Advisory Structure (see section 7). At some point prior to launch the target list will have evolved into a description of the final nominal prelaunch ARIEL target list, however, it can - and should - evolve during the mission if there are compelling scientific reasons. 
Table 1 Example overview of the three tiers of the ARIEL observational strategy

\begin{tabular}{|c|c|c|}
\hline Tier name & Observational strategy & Science case \\
\hline $\begin{array}{l}\text { Reconnaissance } \\
\text { survey } \\
(\sim 1000 \text { targets }) \\
(\sim 30 \% \text { of time })\end{array}$ & $\begin{array}{l}\text { Low spectral resolution } \\
\text { characterisation of entire } \\
\text { sample }\end{array}$ & $\begin{array}{l}\text { - What fraction of planets are covered by clouds? } \\
\text { - What fraction of small planets have still retained } \mathrm{H} / \mathrm{He} \text { ? } \\
\text { - Classification through colour-colour diagrams? } \\
\text { - Constraining/removing degeneracies in the interpretation of } \\
\text { mass-radius diagrams } \\
\text { - Albedo, bulk temperature \& energy balance for a subsample. }\end{array}$ \\
\hline $\begin{array}{l}\text { Deep survey } \\
(\sim 500 \text { targets }) \\
(\sim 60 \% \text { of time })\end{array}$ & $\begin{array}{l}\text { Higher spectral resolution } \\
\text { observations of a } \\
\text { subsample }\end{array}$ & $\begin{array}{l}\text { - Main atmospheric component for small planets } \\
\text { - Chemical abundances of trace gases } \\
\text { - Atmospheric thermal structure (vertical/ horizontal) } \\
\text { - Cloud characterization } \\
\text { - Elemental composition }\end{array}$ \\
\hline $\begin{array}{l}\text { Benchmark } \\
\text { planets } \\
(\sim 50 \text { targets }) \\
(\sim 10 \% \text { of time })\end{array}$ & $\begin{array}{l}\text { Very best planets, } \\
\text { re-observed } \\
\text { multiple times with all } \\
\text { techniques }\end{array}$ & $\begin{array}{l}\text { - Very detailed knowledge of the planetary chemistry and } \\
\text { dynamics } \\
\text { - Weather, spatial \& temporal variability }\end{array}$ \\
\hline
\end{tabular}

\subsubsection{Scientific requirements}

To maximise the science impact achievable by ARIEL, it is needed to access all the molecular species expected to play a key role in the physics and chemistry of the planetary atmospheres. It is also essential to be able to observe warm and hot planets at a wide range of different temperatures (primarily from $\sim 500 \mathrm{~K}$ to $3000 \mathrm{~K}$ ) to probe the differences in composition potentially linked to formation and evolution scenarios. The desired molecular signatures are mainly found in infrared (see Fig. 1 left), however, characterisation of hazes and stellar activity makes it necessary also to cover shorter wavelengths in the optical (see Fig. 1 right). It is required that - after post-processing stellar variability makes a negligible $(<10 \%$ RSS) contribution to the noise budget.

The above considerations lead to a set of concrete science requirements in terms of wavelength coverage, spectral resolving power (R), and required signal to noise (SNR), which can be mapped to specific scientific motivations, as shown in Table 2 .

In addition there are critical requirements on in particular photometric stability, the driver for overall mission design, and in addition also for sky visibility, temporal
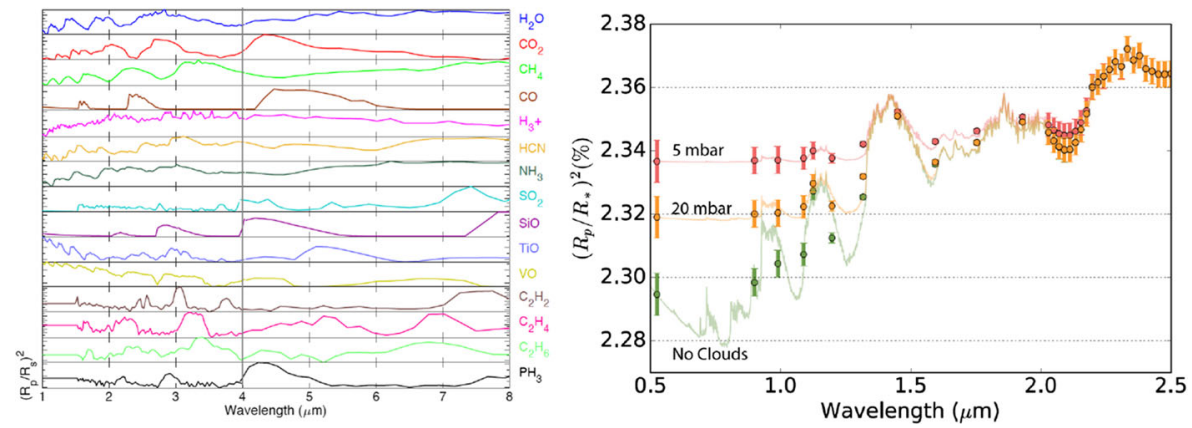

Fig. 1 Left: Molecular signatures in the $2-4 \mu \mathrm{m}$ range with $R=100$, and $4-8 \mu \mathrm{m}$ range with $R=30$. Right: Examples of cloudy and clear-sky spectra, note the large differences in the $0.5-1 \mu \mathrm{m}$ range, this is also the prime range to monitor stellar activity 
Table 2 Required spectral range, spectral resolving power (R), and signal to noise (SNR), mapped to scientific motivations

\begin{tabular}{|c|c|c|c|c|}
\hline \multirow{2}{*}{$\begin{array}{l}\text { Wavelength } \\
\text { range }\end{array}$} & \multicolumn{3}{|c|}{ Required R \& SNR } & \multirow[t]{2}{*}{ Scientific motivation } \\
\hline & Tier 1 & Tier 2 & Tier 3 & \\
\hline $\begin{array}{l}\text { VISPhot } \\
0.5-0.55 \mu \mathrm{m}\end{array}$ & \multicolumn{3}{|c|}{$\begin{array}{l}\text { Integrated band } \\
\text { SNR } \geq 200 \text { on the Stellar SNR } \\
\text { SNR } \geq 7 \text { on the exoplanet (goal) }\end{array}$} & $\begin{array}{l}\text { - Correction stellar activity } \\
\text { (optimised early stars) } \\
\text { - Measurement of planetary albedo } \\
\text { - Detection of Rayleigh } \\
\text { scattering/clouds }\end{array}$ \\
\hline $\begin{array}{l}\text { FGS1 } \\
\quad 0.8-1.0 \mu \mathrm{m}\end{array}$ & \multicolumn{3}{|c|}{$\begin{array}{l}\text { Integrated band } \\
\text { SNR } \geq 200 \text { on the Stellar SNR } \\
\text { SNR } \geq 7 \text { on the exoplanet (goal) }\end{array}$} & $\begin{array}{l}\text { - Correction stellar activity } \\
\text { (optimised late stars) } \\
\text { - Measurement of planetary albedo } \\
\text { - Detection of clouds }\end{array}$ \\
\hline $\begin{array}{l}\text { FGS2 } \\
\quad 1.05-1.2 \mu \mathrm{m}\end{array}$ & \multicolumn{3}{|c|}{$\begin{array}{l}\text { Integrated band } \\
\text { SNR } \geq 200 \text { on the Stellar SNR } \\
\text { SNR } \geq 7 \text { on the exoplanet (goal) }\end{array}$} & $\begin{array}{l}\text { - Correction stellar activity } \\
\text { (optimised late stars) } \\
\text { - Detection of clouds }\end{array}$ \\
\hline $\begin{array}{l}\text { NIRSpec } \\
\quad 1.25-1.95 \mu \mathrm{m}\end{array}$ & $\begin{array}{l}\text { R: } 10 \text { averaged } \\
\text { bands for } \\
1.25-7.8 \mu \mathrm{m} \\
\text { SNR } \geq 7\end{array}$ & $\begin{array}{l}R \geq 10 \\
\operatorname{SNR} \geq 7\end{array}$ & $\begin{array}{l}R \geq 10 \\
S N R \geq 7\end{array}$ & $\begin{array}{l}\text { - Correction stellar activity } \\
\text { (optimised late stars) } \\
\text { - Detection of clouds } \\
\text { - Detection of molecules (esp. TiO. } \\
\text { VO, metal hydrides) } \\
\text { - Measurement of planet temperature } \\
\text { (optimised hot) } \\
\text { - Retrieval of molecular abundances } \\
\text { - Retrieval of vertical and horizontal } \\
\text { thermal structure } \\
\text { - Detection time variability } \\
\text { (weather/cloud distribution) }\end{array}$ \\
\hline $\begin{array}{l}\text { AIRS (Channels } \\
0 \& 1) \\
1.95-7.8 \mu \mathrm{m}\end{array}$ & & $\begin{array}{l}R \geq 50 \text { for } \lambda< \\
\quad 3.9 \mu \mathrm{m} \\
R \geq 15 \text { for } \\
\lambda>3.9 \mu \mathrm{m} \\
\text { SNR } \geq 7\end{array}$ & $\begin{array}{l}\mathrm{R} \geq 100 \text { for } \lambda< \\
3.9 \mu \mathrm{m} ; \\
R \geq 30 \text { for } \\
\lambda>3.9 \mu \mathrm{m} \\
\mathrm{SNR} \geq 7\end{array}$ & $\begin{array}{l}\text { - Detection of atmospheric chemical } \\
\text { components } \\
\text { - Measurement of planet temps. } \\
\text { (optimised warm-hot) } \\
\text { - Retrieval of molecular abundances } \\
\text { - Retrieval of vertical and horizontal } \\
\text { thermal structure } \\
\text { - Detection time variability } \\
\text { (weather/cloud distribution) }\end{array}$ \\
\hline
\end{tabular}

resolution of measurements, limiting cases for targets, and calibration. A summary of these is provided in Table 3.

\section{Remarks:}

Wavelength coverage: Spectral coverage over a broad wavelength range is required not only to cover the wide range of planetary temperatures and molecular/atomic features (the key observables), but also to monitor and remove the variability of the host star. With an instantaneous baseline wavelength coverage spanning over 4 octaves, it is necessary to split the waveband in a series of discrete photometer and spectrometer channels. Baseline and goal requirements have been formulated that prioritise the protection of key wavelength intervals.

Resolving power: The final spectral resolving power, $\mathrm{R}(\lambda / \Delta \lambda)$, and ultimately the spectral resolution actually achieved for any observation, is a trade-off between the 
Table 3 Stability, sky visibility, temporal resolution, limiting targets, and calibration requirements

\begin{tabular}{|c|c|}
\hline Requirement & Value: baseline (goal) \\
\hline Photometric stability & $\begin{array}{l}\text { Stability up to } 10^{-4} \text { required in } 28 \mu \mathrm{Hz} \text { to } 11 / 3.3 \mathrm{mHz} \text { range for bright/faint targets. In } \\
\text { effect, this requires the observations to be photon noise dominated for all potential } \\
\text { targets (within the range defined by the limiting targets). }\end{array}$ \\
\hline $\begin{array}{l}\text { Sky visibility/source } \\
\text { accessibility }\end{array}$ & $\begin{array}{l}30 \% \text { of the sky should be accessible at any one time. The same } 30 \% \text { shall be accessible } \\
\text { over a period of } \sim 10 \mathrm{~h} \text {. The complete sky shall be accessible within a year, a source at } \\
\text { the ecliptic shall be observable for }>30 \% \text { of the mission lifetime. }\end{array}$ \\
\hline $\begin{array}{l}\text { Temporal } \\
\quad \text { resolution/cadence }\end{array}$ & $\begin{array}{l}\leq 90 \mathrm{~s} \text { for stars with } \mathrm{m}_{\mathrm{K}} \leq 6.3, \leq 300 \mathrm{~s} \text { for fainter stars (interval } \leq 1 \mathrm{~s} \text { for FGS1 \& FGS2 } \\
\text { measurements of host star during single transit/occultation). }\end{array}$ \\
\hline $\begin{array}{l}\text { Limiting target: } \\
\text { faintest }\end{array}$ & M5V star with $\mathrm{m}_{\mathrm{K}}=8.8$ (equivalent to GJ 1214). \\
\hline $\begin{array}{l}\text { Limiting target: } \\
\text { brightest }\end{array}$ & K3V star with $m_{K}=3.25$ (equivalent to HD 219134). \\
\hline Calibration & $\begin{array}{l}\text { Absolute flux calibration to } 5 \% \text { (TBC); knowledge of wavelength to } 1 / 3 \text { of the width of } \\
\text { the relevant spectral resolution element; relative photometric accuracy between the } \\
\text { photometric channels of } 100 \mathrm{ppm} \text { (achieved using celestial calibrators). }\end{array}$ \\
\hline
\end{tabular}

desire to resolve as many spectral features as possible and the need to detect these same features at a statistically significant level. It will therefore depend on the brightness of the star, planet/star contrast and the observing time available, as well as the sensitivity of the payload.

Photometric stability: Photometric stability is the critical requirement of the mission. The objective is to observe exoplanets with contrast ratios between the exoplanet and host star of as low as 10 ppm and typically $100 \mathrm{ppm}$. To achieve this with the SNR \& resolving power combinations in the different survey tiers typically requires the co-addition of data taken over a few to - in exceptional cases -tens of transit/occultation events, depending on the characteristics of the individual exoplanet/host star systems. In order that the co-addition itself does not add a systematic noise component to the data, a stability of no more than $100 \mathrm{ppm}$ (see Fig. 5) would be needed in each transit event. This stability is required over a frequency range defined by the timing of the transit event itself, which is known with high accuracy. The maximum timescale is set by the duration of an observation of an exoplanet known today which has a relatively long transit duration: assuming an equal observing time spent in- and out- of transit, this sets the interval to be around $10 \mathrm{~h}$. The minimum relevant timescale is that required to resolve temporally the exoplanet ingress/egress of $\sim 15 \mathrm{~min}$ (GJ436b). These limits together were used to set the baseline frequency intervals; the goal limits are set by the duration of a representative phase curve observation and the shorter cadence time given below.

Sky visibility/source accessibility: The target list will specify a well-defined set of targets, with repeat visits typically required to build up the SNR of individual target spectra. The maximum duration of a visit to a target system would be $\sim 10 \mathrm{~h}$ - the time of the transit itself, plus half that time before and then after the transit. The time between successive transit observations depends on orbital period and scheduling, and could be as little as less than a day, to as long as a few tens of days. In principle, the targets may be in any part of the sky: the satellite needs a large 
field of regard, with minimal constraints on the direction in which it can be pointed (e.g. Earth/Sun pointing restrictions) (Figs. 2 and 3).

Temporal resolution/cadence: Additional constraints on the atmospheric models are provided by knowledge of the shapes as well as the depths of the transit events: the accuracy and reliability with which atmospheric parameters can be derived will depend on both the final SNR of the spectra and on the temporal sampling achieved. One of the shortest ingress periods known to-date is that of GJ 436, a hot Neptune, with a $\sim 15$ min duration. To sample this ingress/egress with at least 10 measurements, the temporal resolution requirement was set to $90 \mathrm{~s}$.

Limiting targets, sizing cases: A set of sizing targets was specified to establish the maximum and minimum stellar flux levels that can be expected over the ARIEL wavelength range. These levels do not preclude the observation of brighter or fainter examples, and were only used to set the mission and payload requirements ("sizing" the mission). At the bright end, the stellar flux can potentially impact on the maximum sampling time for the detector readout, and also on the pointing stability requirements of the satellite itself; at the faint end, the target host stars play an important role in defining the performance requirements for the telescope, instruments and detectors and determines the accuracy of the fine pointing.

Calibration: Calibration (amplitude and wavelength) of spectra can be both relative and absolute. Here absolute calibration is defined as the conversion of

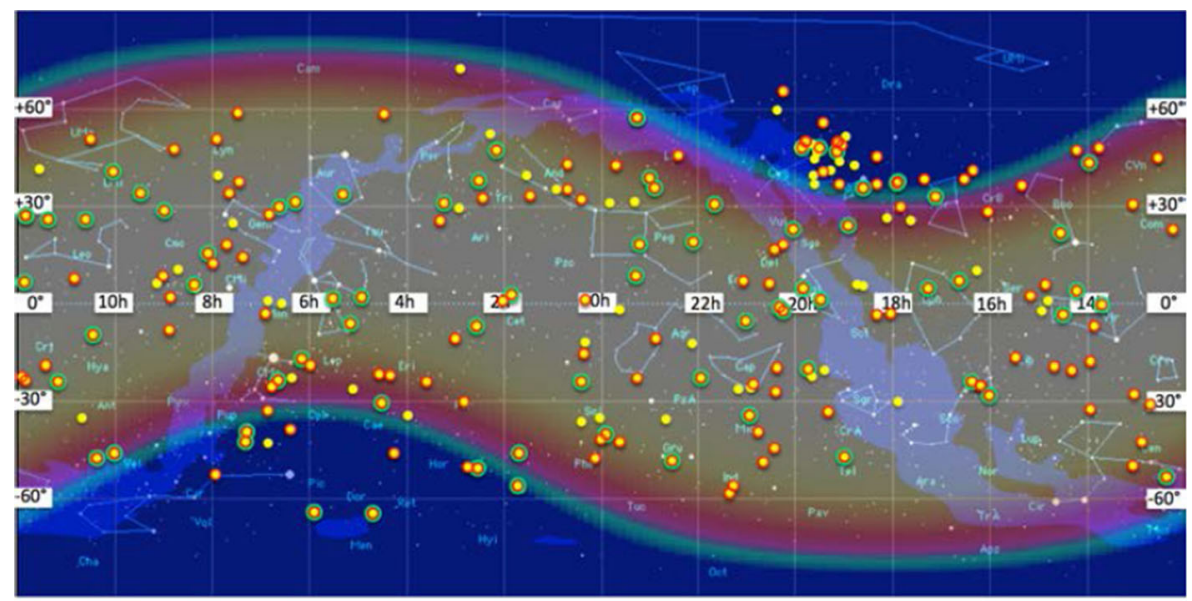

Ariel sky visibility (all known targets)

- Benchmark o Deep Survey

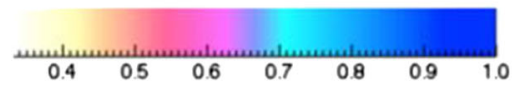

Fig. 2 A plot illustrating the fraction of the year for which a given location in the sky (in equatorial coordinates) is visible to ARIEL, as seen from a representative operational orbit of ARIEL around L2. Orange and green targets are the currently known best targets in term of stellar brightness and planetary parameters (green are the very best, including e.g. 55 Cnc e, HD 189733b, HD 209458 b, GJ 436 b etc.), yellow targets are currently known transiting planets observable by ARIEL 


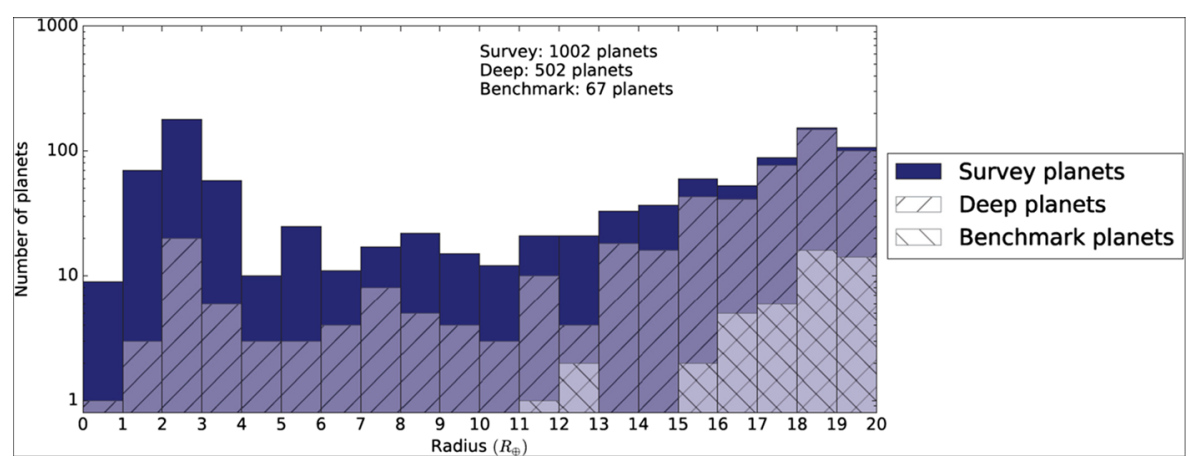

Fig. 3 Overview of an example ARIEL target list, produced by simulations. The graph shows the three tiers Reconnaissance, Deep and Benchmark planets - nested. The current ARIEL design enables the observation of $\sim 1000$ planets during the baseline mission lifetime which exceeds the mission requirements. It is clear that this example list of planets will change over the years depending on the new exoplanet discoveries

the recorded signals from the instrument into physical units and comparison to some standard system. Absolute knowledge of the target flux is not necessary for the detection of spectral features or recovery of planetary atmosphere models as models typically rely on line-to-continuum ratios and, importantly, knowledge of the relative variation of the continuum as a function of wavelength. Relative calibration between the various channels of the instrument is however critical.

\subsubsection{The target list}

Establishing the pool of potential targets from which the actual ARIEL target list would ultimately be drawn has been a key activity of the science team during the study. A static radiometric model was used to determine the signal-to-noise achievable for transit and occultation observations of individual and samples of targets. Based on conservative assumptions of the achievable mission/instrument performance, as appropriate to study phase, it was shown that the requirements on the numbers and diversity of targets in the ARIEL core sample could readily be met [5].

Known targets already make up a diverse sample of exoplanets and host stars, with a pool from which the targets could be drawn that will continue to increase in breadth and number with the results of ongoing ground-based and space-based surveys as well as future planned space missions, in particular by the NASA TESS mission, to be launched in early 2018 [6].

Efficient scheduling of the large number of individual, time-critical transit events needed to complete observations of the complete sample - would be essential to the success of the mission. Tools has been developed to generate optimized long-term mission observing plans using ephemerides of exoplanet targets, taking into account mission-level constraints such as data down-links, satellite station-keeping, calibration needs and target visibility. Results suggest that it will be possible to schedule a maximum of around $85 \%$ of the total time depending on the exact details of the list of targets. The resulting gaps would to be filled by extending pre- and post-transit durations, and possibly by science beyond exoplanet science.

Further details on the scheduling activities can be found in $[7,8]$. 


\section{Mission requirements}

\subsection{Flow down to mission requirements - document tree}

To fulfill the science objectives detailed in Section 2, the science requirements were translated into system level engineering requirements in the Mission Requirements Document (MRD). The main requirements in the MRD can be grouped into 3 categories and are summarised in the following subsections: mission, observing and performance requirements. Other requirements are also included and relate to defining standards and coordinate systems, high level ground segment requirements, spacecraft autonomy and reliability requirements and requirements derived from programmatic constraints.

\subsection{Mission requirements}

The mission design must be compatible with an Ariane 6 launch from Kourou in 2026. The baseline injection strategy is to place the ARIEL S/C in an eclipse-free (Earth and Moon) direct transfer trajectory to the Sun-Earth L2 point. The science operations orbit is an eclipse-free orbit around the Sun-Earth L2 point, with an amplitude no larger than 1.5 million $\mathrm{km}$ (Sun-S/C-Earth angle $\leq 45^{\circ}$ ).

In addition to this baseline launch strategy, the mission design is kept compatible with a few other alternatives:

- Launch into a LEO circular parking orbit before injection into a transfer trajectory to L2.

- Dual launch (making use of the higher A6 performance compared to Soyuz) with another passenger. In this case, both injection orbits are also possible (direct transfer to L2 or circular parking orbit).

The mission design has a number of distinct phases (timeframe for completion of activity given in brackets): Launch campaign (L); Launch and early operations phase (LEOP $-\mathrm{L}+2$ days maximum); Transfer phase + Commissioning phase (initiated during the transfer phase) $(\mathrm{L}+3$ months); Performance verification and science

Table 4 ARIEL delta-V requirements

\begin{tabular}{ll}
\hline Manoeuvre & Delta V [m/s] \\
\hline Transfer to L2 & 47.32 \\
Station keeping in L2 & 22.18 \\
Decommissioning & 10 \\
Soyuz to A5 compatibility & 23.44 \\
Margin & 10 \\
Contingency & 15 \\
Total & 127.94 \\
\hline
\end{tabular}

Numbers are based on a Soyuz launch. A correction is included to cover the different injection accuracies between Soyuz and Ariane 5. Predictions of the Ariane 6 injection accuracy are not yet available, but expected to be at least as good as that of Ariane 5 
demonstration phase ( $\mathrm{L}+6$ months); Nominal (and extended) science operations phase; decommissioning phase; Post-operations phase (end of in-flight operations +2 years).

The mission lifetime (from launcher separation till the end of the nominal science operations phase) is set to a duration of 4 years based on the science requirements, with an additional 2 years extension as a goal which in turn drives the amount of $\mathrm{S} / \mathrm{C}$ consumables and the design of radiation-sensitive units.

The delta-V requirements are summarized in Table 4.

\subsection{Observing requirements}

The overall observation efficiency (or duty cycle) of the ARIEL S/C during the science operation phases is required to be $\geq 85 \%$. The remaining $15 \%$ included outages such as orbital manoeuvres, slews between targets, safe modes etc. An average observation $(7.7 \mathrm{~h})$ and angle between consecutive science targets (70 degrees) were defined to determine the speed and propellant requirements for the slews.

Requirements on sky accessibility were taken directly from the science requirement (see section 2.2.2). This translates into requirements for the spacecraft to be able to do a full $360^{\circ}$ and $\mathrm{a} \pm 25^{\circ}$ rotation around the spacecraft's yaw and pitch axes respectively and to observe a target from any of those attitudes.

The faintest and brightest targets that ARIEL is designed to observe are defined in Table 3. Their spectral energy distributions (SEDs) are shown in Fig. 4.

\subsection{Performance requirements}

Requirements on wavelength and resolving power are detailed in section 2.2.2.

The required telescope FoV is small, since only a single star would be observed at any given time. Additional margins are required to take into account the pointing errors and operational planning for target acquisition and centring, and result in a FoV of the order of a few tens of arcsec.

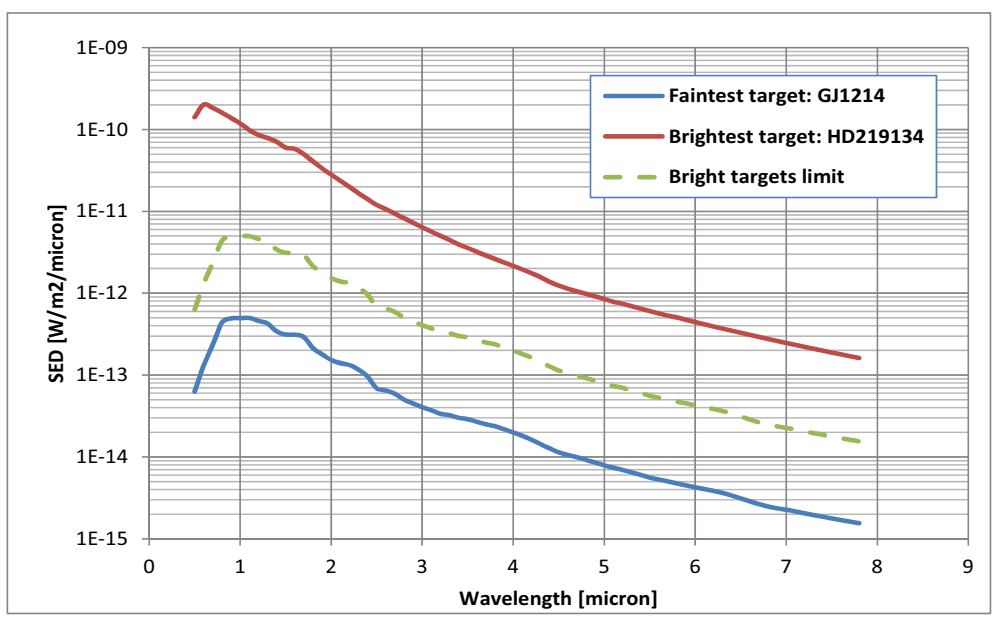

Fig. 4 Faintest and brightest host stars SEDs 
Since the telescope effective area $\left(A_{\text {eff }}\right)$, the throughput $(\eta)$ of the optics and the quantum efficiency $(\mathrm{QE})$ of the detectors all have an equivalent effect on the final SNR achieved, they are grouped into a single figure of merit. The product $\mathrm{A}_{\mathrm{eff}} \mathrm{x} \eta \mathrm{x} \mathrm{QE}$ is required to be:

$-\geq 0.6 \times 40 \% \times 55 \%=0.132\left[\mathrm{~m}^{2} . \mathrm{e}-/\right.$ photons $]$ above $1.95 \mu \mathrm{m}$.

$-\quad \geq 0.6 \times 50 \% \times 55 \%=0.165\left[\mathrm{~m}^{2}\right.$.e-/photons $]$ below.

This figure of merit is defined as an average value in each channel. The minimum inchannel figure of merit is required to be no less than $80 \%$ of the value above, while a
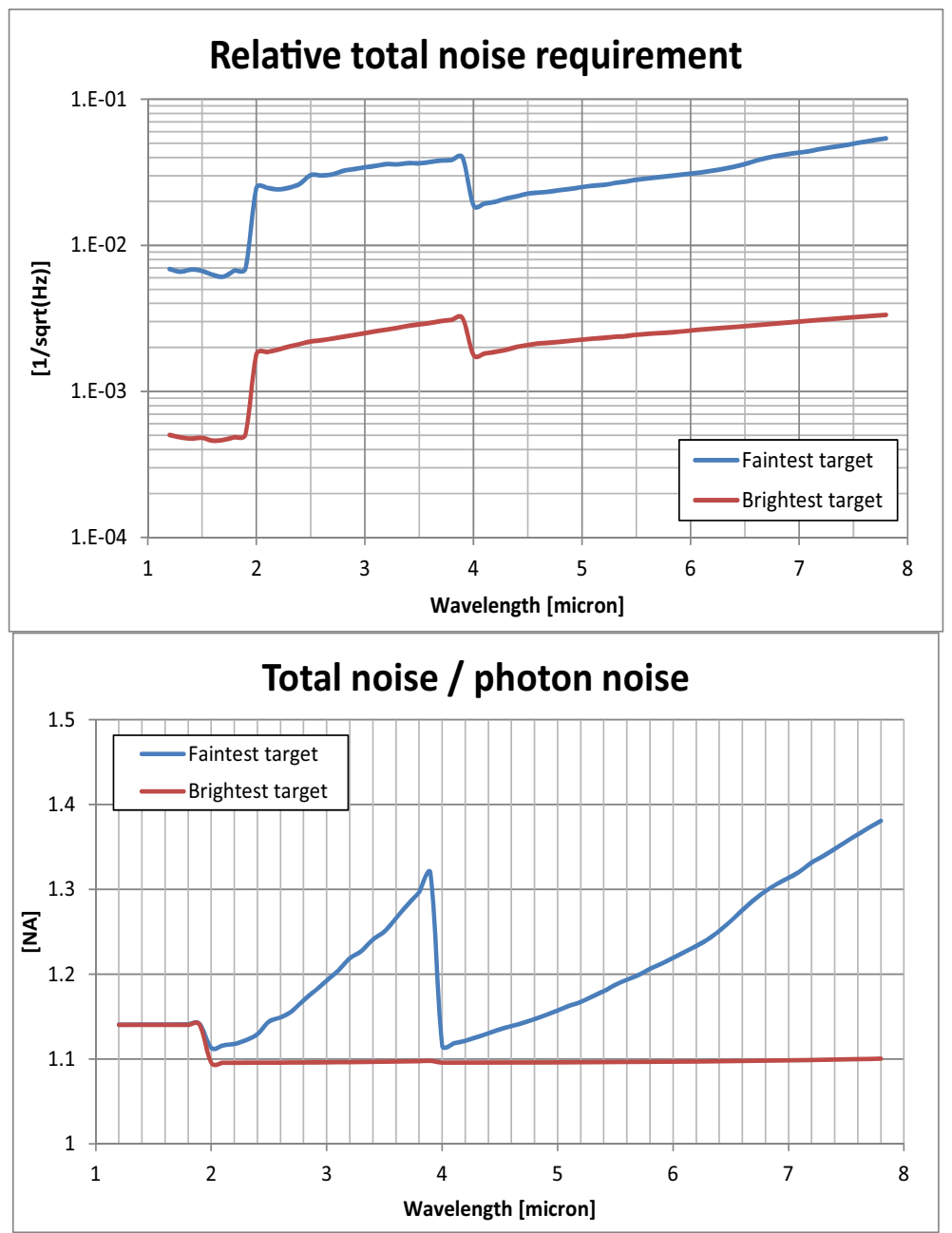

Fig. 5 Top: ARIEL relative total noise PSD requirement per spectral bin in all spectroscopic channels. It is the ratio between the total noise (excess system noise plus photon noise) and the stellar flux. Bottom: Ratio between the total noise and the astronomical photon noise. This shows the system is close to the photon noise limit, with the excess noise representing no more than $\sim 10 \%$ of the photon noise for bright sources, and no more than $40 \%$ of the photon noise for faint sources 
reduction down to $50 \%$ out-of-channel (i.e. in the overlap regions between all channels) is acceptable, meaning that some significant performance is achieved continuously throughout the entire wavelength band.

The most important performance requirement is the system-level noise. This is defined post-processing, and takes into account all noise sources, including photometric variations (e.g. due to the pointing stability, the thermal background stability etc.) in the frequency band given in Table 3. It is defined as an allowable excess variance on top of the fundamental astronomical noise set by the stellar and the zodiacal background shot noise contributions (photon noise) (Fig. 5 and Tab. 5).

\section{Mission profile and operations}

\subsection{Launcher, launch window and orbit selection}

The proposed science operations orbit is an eclipse-free (Earth and Moon) large amplitude orbit around the Sun-Earth L2 point, offering a very stable environment (for thermal, power and communication purposes), combined with a very large instantaneous field of regard. This orbit offers several other advantages: the radiation environment in L2 is much more benign compared to LEO orbits, and it is not too distant from the Earth, allowing a simple communication strategy and communication subsystem design (Fig. 6).

The baseline launch strategy consists of a Ariane 62 or 64 launch from Kourou into a direct transfer orbit to L2. The transfer and insertion into L2 are designed to be propellantfree, however opportunities at day 2, 5 and 10 after launch are available to correct for launcher dispersion and perigee velocity errors. Due to the still unknown Ariane 6 launcher performance, the Soyuz figures are used for performance assessment. The Soyuz provides an injected mass performance of about $2.2 \mathrm{t}$ towards L2 (at least $3 \mathrm{t}$ expected with A6). The Ariel launch mass (wet mass + adapter) is $\sim 1.3 \mathrm{t}$, including the typical $20 \%$ system level margin. This leaves about $900 \mathrm{~kg}$ unused (even more expected with A6). This, combined with the large volume margin of Ariel inside the A6 fairing, should also allow to use a dual launch configuration, with a companion spacecraft on top of a dual launch adapter (and ARIEL underneath to protect its cryogenic payload from direct Sun illumination during LEOP). Aside from the direct transfer, a launch into an intermediate circular parking orbit is still being considered as well.

Table 5 As in Fig. 5, but for ARIEL's photometric channels

\begin{tabular}{llll}
\hline & & $\begin{array}{l}\text { Relative total noise PSD } \\
\text { requirement }[1 / \sqrt{\mathrm{Hz}}]\end{array}$ & $\begin{array}{l}\text { Total noise / astronomical } \\
\text { photon noise [NA] }\end{array}$ \\
\hline Faint target & VISPhot & $4.18 \mathrm{E}-2$ & 1.24 \\
& FGS1 & $6.04 \mathrm{E}-3$ & 1.14 \\
Bright target & FGS2 & $6.17 \mathrm{E}-3$ & 1.14 \\
& VISPhot & $1.05 \mathrm{E}-3$ & 1.14 \\
& FGS1 & $5.14 \mathrm{E}-4$ & 1.14 \\
& FGS2 & $6.62 \mathrm{E}-4$ & 1.14 \\
\hline
\end{tabular}




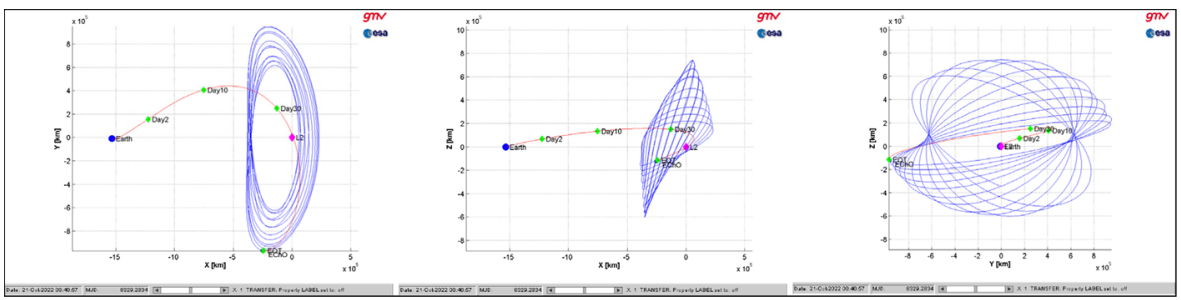

Fig. 6 Example of Ariel's orbit around L2

The daily launch window is greatly influenced by the illumination and thermal constraints (to minimise direct Sun illumination of the cryogenic PLM). Closures of the launch window occur near the solstices as a consequence of the geometry. As a result, two launch seasons exist where daily launch windows of up to $90 \mathrm{~min}$ are common, except for around the equinoxes when eclipse avoidance further constrains the launch window. More generally, the main conditions that were used to constrain the launch window are:

- No eclipses allowed during the mission lifetime (including the transfer and the 2 year mission extension), for thermal and power generation stability.

- Propellant-free injection into L2 to minimise propellant mass.

- An orbit amplitude around $\mathrm{L} 2 \leq 1.5$ million $\mathrm{km}$ to minimise the $\mathrm{S} / \mathrm{C}$ to Earth distance for the communications subsystem, leading to a Sun-S/C-Earth angle $\leq 45^{\circ}$.

- Due to the cryogenic nature of the ARIEL PLM placed on top of the SVM and the mid-day launch windows (i.e. facing the Sun), additional constraints are placed on the launch window to minimise the duration during which the PLM is exposed to direct Sun illumination.

Concerning this last pointhowever, the MSR concluded that the impacts of this Sun illumination (modifications of materials and coatings on the PLM are needed to survive this phase, with an impact on the cryogenic performance, plus the need to test and qualify the PLM against this environmental condition) are severe and should be mitigated by investigating alternative scenarios where this constraint is no longer present. Two possibilities are being envisaged:

- The dual launch scenario where ARIEL is protected from the Sun under the dual launch adapter.

- The addition of an ejectable PLM thermal cover in the S/C design.

To enable this second option, the transfer trajectory to L2 has to be slightly modified to ensure the thermal cover does not add to the debris population in Earth orbit. This can be achieved by ensuring the cover returns to Earth and burns in the atmosphere, or by ejecting it on an Earth escape trajectory. Both scenarios will require an additional manoeuvre to ensure ARIEL arrives at L2 with a small DV penalty that will need to be added in Table 4. 


\subsection{Operations}

The ARIEL mission is planned with a short 2 day LEOP, followed by a 90 day Commissioning Phase. The orbit transfer to L2 occurs within this Commissioning Phase. After completion of the LEOP activities the first Transfer Correction Manoeuvre (TCM) would be executed. Two further TCMs are foreseen with nominal arrival at operational orbit within 30 days of launch. Initial commissioning activities would be carried out during the transfer phase with completion of commissioning envisaged for 90 days after launch. The Commissioning Phase would be followed by another 90 days of instrument performance verification and science demonstration phase before the nominal science operations can start. Standard tasks to be undertaken during these phases would include commissioning of all the $\mathrm{S} / \mathrm{C}$ subsystems and verification of the payload performance and calibration.

The nominal science observing strategy for ARIEL is such that the average observation of a single science target will be in the order of $7.7 \mathrm{~h}$ with a separation of 70 degrees from the previous and next science target. The maximum observation time is in the order of $10 \mathrm{~h}$. The required overall observation efficiency is $\geq 85 \%$. Outages in this observation time are driven mainly by station keeping manoeuvres (approximately every 30 days), slews between targets and an allocation for safe modes.

The ARIEL mission will be operated out of a single ESA Mission Operations Centre (MOC) located at ESOC in Darmstadt. X-Band will be used for telecommand uplink, real-time housekeeping downlink and the high rate stored housekeeping telemetry and science data telemetry downlink. Use of the ESA ESTRACK $35 \mathrm{~m}$ antenna network is planned to support these activities.

For routine operations the ARIEL mission is foreseen to be operated in a highly autonomous way. The ARIEL mission on board capability is foreseen to handle simplified commanding for execution of slews between observations based on requested target position; reaction wheel momentum management will be controlled on board and all operations executed on board from preloaded mission timelines. This approach reduces the need for ground-based definition of the slew profiles and momentum management. The mission timelines are uplinked on a periodic basis (e.g. weekly) and the shortened pass durations ( $\sim 14 \mathrm{~h}$ per week split in 3 passes) are primarily used for downlink of the science data and collection of tracking data needed for orbit determination. The orbit control is anticipated to be of a predictive nature allowing the mission to apply rule based planning for engineering windows for platform operations, this allows for the remaining science planning to be done on a long-term basis by the SOC. Thanks to a steerable Medium Gain Antenna (MGA), the ground station passes can be scheduled independently of the science observations, and these passes do not affect the observation efficiency as science observations and ground contact can be performed in parallel.

The ARIEL mission will be designed for a nominal 4 year lifetime, resulting in a 3.5 year nominal science operations phase (after deduction of the commissioning and science performance verifications phases). Mission consumables are sized for an additional 2 year extended science operations phase. The ARIEL mission would be terminated with a de-orbiting and mission disposal phase where ARIEL would be removed from the L2 orbit and put in a heliocentric orbit with a very low probability for a return to Earth. This final decommissioning/disposal phase is foreseen to last up to 90 days. 


\subsection{Ground segment and science operations}

The ARIEL Ground Segment (GS) provides the means and resources with which to manage and control the mission via telecommands, to receive and process the telemetry from the satellite, and to produce, disseminate and archive the generated products. Responsibility for and provision of the ARIEL GS is split between ESA and a nationally-funded Instrument Operations and Science Data Centre (IOSDC), with the ESA part comprising the MOC, the tracking station network (ground stations), and the Science Operations Centre (SOC) located at ESAC near Madrid. A schematic view of the operational interfaces for the ARIEL mission is presented in Fig. 7.

The core of the scientific mission planning consists in generating and maintaining a long-term observation plan of the ARIEL surveys including the needed calibration observations, based on the list of target observation and mission constraints. Inputs to the target list will be solicited from the wider scientific community (e.g. through whitepapers, meetings, and other mechanisms) that will be kept informed about the status of the target list.

The rule-based inputs of MOC constraints and the independency of ground station bookings lead to a highly simplified and optimized monthly mission planning cycle. An automatic planning tool developed by the IOSDC based on a figure-of-merit optimization process would deliver the initial long-term plan before launch based on the selected target list by the ARIEL science team, and updates in regular intervals as new targets may be added. The target list would be converted into a sequence of observations with high scheduling efficiency to maximize the scientific return. These observation sequences consisting of scheduled pointings and payload configurations for each observation are sent from SOC to MOC as part of payload and spacecraft commands in the mission timeline for uplink to the spacecraft.

Data products from raw telemetry to the extracted target (star + planet) spectra, free of instrumental signatures, would be produced at the SOC using processing pipelines developed by the IOSDC and delivered to SOC in form of virtual machines for autonomous operation. The extraction of the final exoplanet spectra from the stacked target observations throughout the mission is under the responsibility of the IOSDC using state-of-the-art tools. These core science products would be delivered to the SOC for ingestion into the ARIEL archive hosted at ESAC. Re-processing of the data is foreseen when new calibration data would become available or new insights into processing steps require modifications to the pipeline modules. Cadence is currently foreseen to be approximately once per year.

The ARIEL archive will be developed by ESA and would be the repository for all data associated with all phases of the mission, fulfilling the role of mission database and science data archive. Before launch it would be used for instrument-level testing and on-ground calibration. During operations it would be used to store raw telemetry, processed science data products, calibration products as well as relevant support data. The higher-level data products together with the relevant pipelines and calibration data would be made available to the scientific community through the archive based on the agreed data release policy (see section 7), with user support provided by SOC in close collaboration with the IOSDC. This will allow the scientific users to re-process the science data using their own preferred tools for exoplanet science if they so wish. Post- 


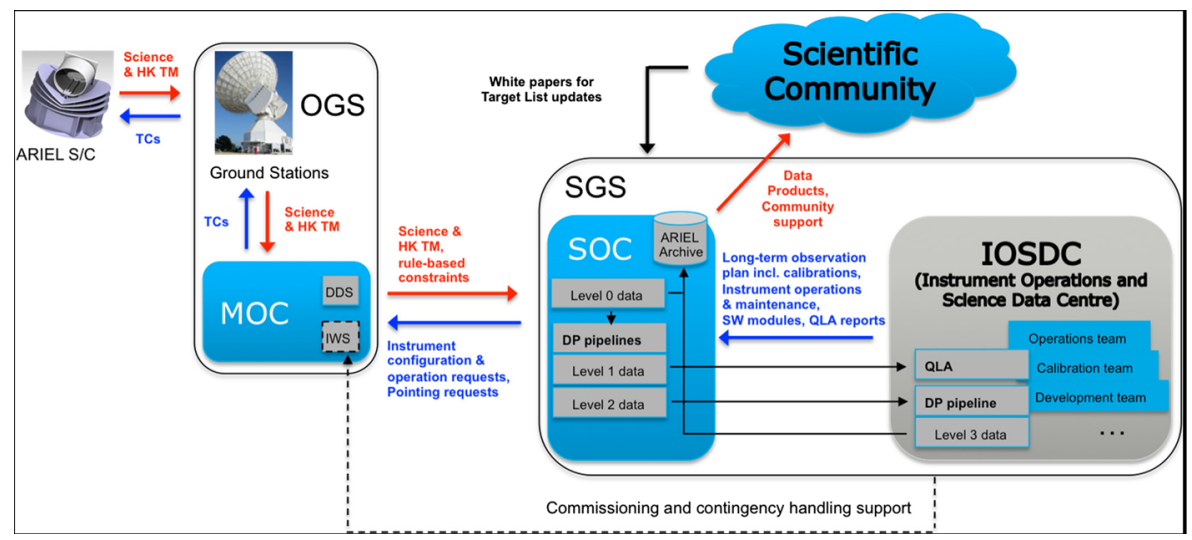

Fig. 7 ARIEL Ground Segment interfaces and data flow. SOC as part of the Science Ground Segment (SGS) is the nominal point of contact to MOC during in-orbit operations, exceptions include the commissioning phase and the case of contingency handling, where a direct link between IOSDC and MOC can be established through the instrument workstation (IWS). Comunication paths (commanding and data flow) are indicated by arrows. The raw telemetry (science and housekeeping TM) is distributed to the SGS via the Data Disposition System (DDS) at MOC, which is managing the private communication to the ARIEL spacecraft through the ground stations as part of the Operational Ground Segmant (OGS). The operational location of the data processing pipelines up to the final exoplanet spectra (Level 3 data), archiving and interface to the scientific community are indicated as well

operations and following the archiving phase, final versions of all calibration files, data products and support data as well as processing software/pipelines including user documentation would reside in the archive as part of the long-term science data preservation plan of the science data archives at ESAC.

\section{Spacecraft design}

\subsection{Design drivers}

The most stringent requirements applicable to the ARIEL mission are:

- Wavelength range.

- Near photon noise limited system.

- Photometric stability of up to $\sim 10^{-4}$ or better (depending on the target).

These requirements have the following system level impacts:

- The noise threshold over the wavelength range means low read out noise and low dark current Mercury-Cadmium-Telluride ( $\mathrm{HgCdTe})$ detectors operated at a temperature as low as $\sim 40 \mathrm{~K}$ (for AIRS channel \#1) are required. This necessitates an active cryo-cooler.

- The instrument box should have a temperature of $\sim 55 \mathrm{~K}$ to reduce its thermal background and limit its variations, as well as operate the detector cold Front End Electronics (FEE). This can be achieved by a passive cooling system. 
- Similarly, the telescope also needs to be at a temperature of $\leq 70 \mathrm{~K}$ (or less to ease the thermal background stability requirement) to reduce its thermal background. For simplicity of the thermal/cryogenic design of the PLM, the complete PLM (optical bench, instrument box and telescope) is passively cooled to $55 \mathrm{~K}$ to minimize the number of different temperature stages.

- A small Pointing Drift Error (PDE) of the instrument Line of Sight is needed, down to $\sim 100$ mas over $10 \mathrm{~h}$ for observations of up to $90 \mathrm{~s}$ (half-angle, radial, 3 sigma). The pointing requirements impose the adoption of a Fine Guidance Sensor (FGS), used in the closed control loop of the Attitude and Orbit Control System (AOCS) of the spacecraft..

\subsection{Instrumentation}

This section presents a brief summary of the ARIEL payload design as baselined by the payload Consortium.

The science case (see section 2.2), calls for medium resolution near and mid infrared spectroscopy and parallel photometry in the visible and near infrared with high accuracy and stability.

The baseline payload consists of a PLM containing a telescope assembly (operating below $70 \mathrm{~K}$ ), an optical bench carrying the cold instruments (operating at $55 \mathrm{~K}$ ) and a radiator, V-groove shields for passive cooling and an active cryocooler system (specifically for cooling the AIRS channel\#1 NEOcam detector between $42 \mathrm{~K}$ and $35 \mathrm{~K}$ ), and the warm payload electronics units accommodated on the SVM. The payload architecture is shown in Fig. 8.

The prime science payload for ARIEL is a broadband, low resolution infrared spectrometer operating between 1.95 and $7.8 \mu \mathrm{m}$ (AIRS, see Table 6). This is a single module that incorporates two channels covering the 1.95-3.9 $\mu \mathrm{m}$ and 3.9-7.8 $\mu \mathrm{m}$ ranges. Wavelength splitting between the two channels is achieved by a dichroic mirror. For wavelengths shorter than $3.9 \mu \mathrm{m}$ a resolution of 100 is required, and 30 for longer wavelengths.

The visible/near infrared photometer and spectrophotometer comprises four channels: two wide band channels which also serve as the fine pointing system (FGS) operating between $0.8-1.0$ and 1.05 and $1.2 \mu \mathrm{m}$, a near infrared spectrometer operating
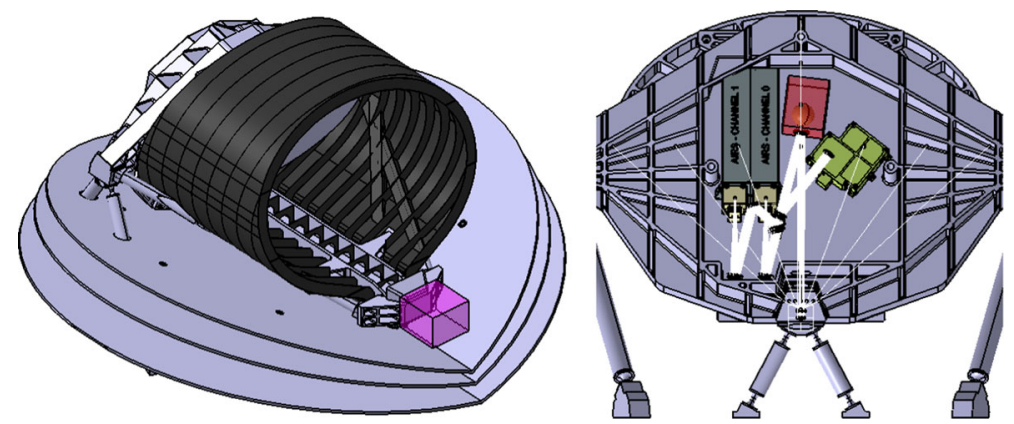

Fig. 8 ARIEL PLM. Top view (left) and back view (right) with the radiator removed, showing the different instrument channels 
Table 6 ARIEL instrument suite

\begin{tabular}{lll}
\hline Channel Name & Wavelength $(\mu \mathrm{m})$ & Resolving power \\
\hline VisPhot & $0.5-0.55$ & Photometer \\
FGS-1 & $0.8-1.0$ & Photometer \\
FGS-2 & $1.05-1.2$ & Photometer \\
NIRSpec & $1.25-1.95$ & $R \geq 10$ \\
AIRS-Channel \#0 & $1.95-3.9$ & $\mathrm{R} \geq 100$ \\
AIRS-Channel \#1 & $3.9-7.8$ & $\mathrm{R} \geq 30$ \\
\hline
\end{tabular}

at $1.25-1.9 \mu \mathrm{m}$ and a visible photometer at $0.5-0.55 \mu \mathrm{m}$. The FGS's main task is to provide the fine pointing knowledge to the satellite, but it will also provide high precision photometry of the target for science. In particular, the data from the FGS will be used for de-trending pointing jitter effects in the science data and for other noise reduction data analysis on ground and to ensure that the atmospheric retrieval can be made without confusion.

Both instruments are fed by a common optics module including dichroic mirrors to separate the various channels.

Current visible and near-infrared detector technology, employed in the photometer, spectrophotometer and FGS channels, requires operating temperatures below $55 \mathrm{~K}$, which in the baseline design can be achieved passively.

To cover with a high quantum efficiency the full wavelength band range of the AIRS system, two HgCdTe HAWAII-1RG detectors from Teledyne are baselined. The first one has a cut-off of $5.3 \mathrm{um}$ and the second one was developed for the NEOCam mission with a cut-off around $10-11 \mu \mathrm{m}$. This NEOcam detector will be cooled actively by a JT $\mathrm{Ne}$ cooler to a temperature of $\leq 42 \mathrm{~K}$. Potential alternative European detectors for the AIRS instrument are under development and show some promising results. For the FGS, the HAWAII-1RG detector is also considered as the baseline while the ALFA (Astronomy Large Format Array) is envisaged as a potential European alternative. All detectors are coupled to cold Front End Electronics (FEE) low-power dissipation to control the detector and insure the analogue-to-digital conversion.

The telescope is an un-obscured, off-axis Cassegrain all Aluminium telescope with an elliptical $1.1 \mathrm{~m} \times 0.7 \mathrm{~m}$ primary mirror. It is foreseen that the secondary mirror will be equipped with a re-focussing mechanism. A third powered mirror collimates the beam before it is passed to the instruments. The telescope is accommodated horizontally on the PLM (see Fig. 8). The image quality of the telescope system is planned to be diffraction limited at wavelengths $\leq 3$ um over a field of view (full angle) of 30 arcsec. The overall envelope of the optics is $1400 \mathrm{~mm} \times 950 \mathrm{~mm} \times 1200 \mathrm{~mm}$.

The overall payload mass allocation is $460 \mathrm{~kg}$ including margins, of which $39 \mathrm{~kg}$ are for the warm units, $90 \mathrm{~kg}$ for the V-grooves and PLM support struts, $300 \mathrm{~kg}$ for the telescope subsystem and $31 \mathrm{~kg}$ for the cold units on the optical bench including cryoharness. The power allocation for all warm units during operations is $140 \mathrm{~W}$. It is expected that the instruments will generate, during routine operations, 25 Gbits per day of science data. During commissioning or observations of specific (bright) science targets peak data rates of a factor of 20 higher may be expected. 

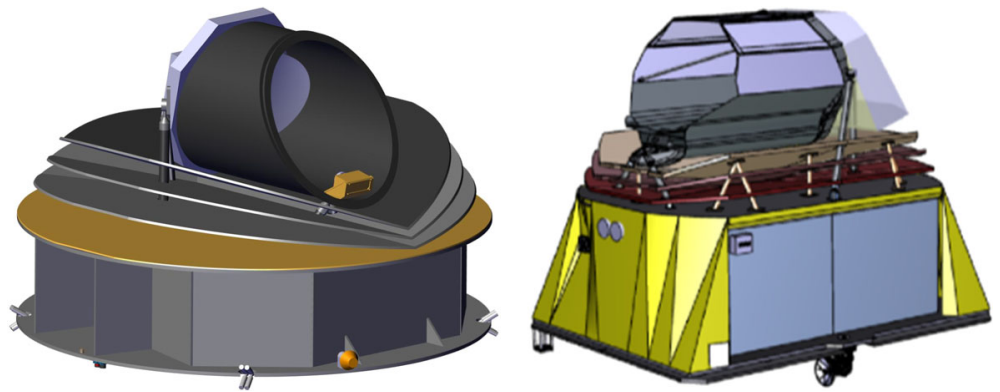

Fig. 9 Industrial S/C designs (courtesy of TAS and ADS) with alternative PLM designs compared to the Consortium baseline PLM

\subsection{S/C platform}

\subsubsection{Structures, configuration and thermal}

The spacecraft is designed in a modular way, with the SVM upper panel acting as a clear physical separation between the SVM and PLM (see Fig. 9). This will simplify the procurement and Assembly, Integration and Verification (AIV) approach by allowing both modules to be procured and tested in parallel and independently.

The SVM structural design differs in shape and size between the two industrial contractors, but uses a common and standard approach, based on a central cylinder or cone, side walls and shear panels, and bottom and top panels (see Fig. 10). The central cylinder/cone is the main structural elements that carries the launch loads and interfaces with the LV adapter at its bottom end, while holding the propellant tank(s) inside. All other $\mathrm{S} / \mathrm{C}$ units are accommodated on the side walls. The SVM also carries the warm payload elements (cryo-cooler, ICU and FCU). The SVM is thermally controlled at $\sim 20{ }^{\circ} \mathrm{C}$ for nominal operations of all the $\mathrm{S} / \mathrm{C}$ subsystem units. This is achieved through dedicated heaters where required and some radiator areas are available on SVM walls that are constantly looking towards the cold sky.

The overall configuration of the $\mathrm{S} / \mathrm{C}$ is driven by the thermal requirements and the accessible sky requirements. The SVM upper panel shape and size drives the design of

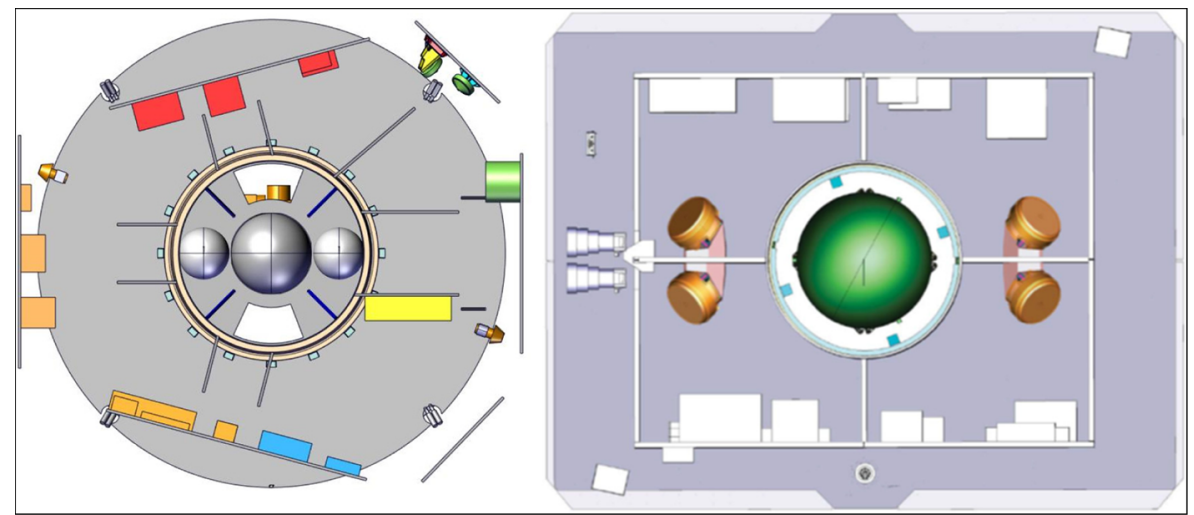

Fig. 10 Inside view of industrial SVM designs (courtesy of TAS and ADS) 
the PLM thermal shield assembly following the required cut angles defined in section 3.3, while it also acts as Sun shield (or V-groove \#0) for the PLM.

\subsubsection{Attitude and orbit control subsystem (AOCS) and propulsion}

Note that all pointing errors detailed in this section are half cone angles expressed with a $99.7 \%$ confidence level.

The Attitude and Orbit Control Subsystem (AOCS) provides control of the S/C attitude and of the telescope and instrument Line of Sight (LoS). The AOCS requirements are split into 2 main pointing modes: a coarse pointing mode and a fine pointing mode.

The coarse pointing mode is achieved by only using the AOCS units accommodated in the SVM, with the star tracker as the main sensor and the reaction wheels as the sole actuactors (i.e. without the FGS in the control loop). This mode is used to slew between target stars. The resulting coarse Absolute Performance Error (APE) of 8" across the LoS of the FGS channels is included in the sizing of the FGS Field of View (FoV). As such, this mode ensures the FGS can acquire the target star within its FoV, to then successfully transition to the fine pointing mode.

The fine pointing mode is the precise pointing mode that will be used during observations of all science targets. It is achieved with the FGS in the control loop to provide fine pointing knowledge around the 2 axes across the LoS of the instruments, hybridised with the star trackers for a coarse determination of the attitude around the 3rd roll axis (pointing errors around the roll axis of the telescope/instruments are less constrained since ARIEL is observing a single target at a time in the centre of the FoV). The main fine pointing requirements achieved by the system across the instruments LoS are:

- Fine APE $\leq 1$ "

- Relative Performance Error (RPE) $\leq 200$ mas up to $90 \mathrm{~s}$

- Performance Drift Error (PDE) $\leq 100$ mas up to $10 \mathrm{~h}$ for integrations of $90 \mathrm{~s}$

These requirements are applicable when observing bright targets. This is because the brighter the target is, the larger the photometric variation induced by a pointing variation is. As a corollary, for fainter targets, these pointing requirements are more relaxed (see Fig. 5). This benefits the AOCS subsystem, since the FGS centroiding error increases slightly with a lower input flux. The bright target pointing requirements given above are to be achieved with a specification on the FGS measurement error $\leq 20$ mas at $10 \mathrm{~Hz}$.

Similarly to the coarse APE and the sizing of the FGS FoV, the fine APE is taken into account in the sizing of the entrance slits of both AIRS channels.

The RPE contains all the high frequency jitter terms. Most of this jitter is unresolved, because the detector read out rates are slower than most sources of high frequency jitter (FGS error at $10 \mathrm{~Hz}$, reaction wheel and cryo-cooler micro-vibrations in the $10 \mathrm{~s}$ to $100 \mathrm{~s} \mathrm{~Hz}$ range). This jitter induces a small blur of the large system PSF (driven by the telescope diffraction limit at approximately $3 \mu \mathrm{m}$ ), but does not result in photometric variations in the frequency range of the science observations.

The PDE is the most important pointing requirement, as it translates directly into photometric variations that add to the instrument noise budget. As the PSF drifts on the 
focal plane detectors, the number of photons incident on the individual pixels will vary. This photometric variation is taken into account in the instrument noise and photometric stability budget. Since the PDE applies to very low frequencies (minutes to hours), it is more easily met than the RPE. The main phenomena that translate into attitude drifts in this frequency range are thermo-elastic errors due to changes in attitude of the $\mathrm{S} / \mathrm{C}$ between targets (and therefore changes in attitude with respect to the Sun leading to different thermal loads on the S/C) and the Solar Radiation Pressure (SRP). In the case of ARIEL the SRP is more than one order of magnitude lower than that of typical astrophysics missions with telescopes that are accommodated vertically (e.g. Herschel or Euclid). This is because the horizontal accommodation of the PLM on the SVM allows for a well-balanced $\mathrm{S} / \mathrm{C}$ which minimises the torque that originates from the misalignment between the centre of gravity of the S/C and the centre of pressure of the SRP. Any drifts occurring from thermo-elastic effects and the SRP are in any case seen by the FGS as it is operating at a much higher frequency, and are therefore corrected by the AOCS subsystem.

While these fine pointing requirements impose the need for the FGS as a fine sensor in addition to the star trackers, they can still be met with reaction wheels as the sole actuators (i.e. no need for a fine pointing actuation system). To achieve this, the reaction wheels need to be operated in a narrow angular speed range, away from any peak vibration mode and away from any possible amplification frequency of the S/C structure (first modes, in the tens of $\mathrm{Hz}$ ). This derives into the need to very frequently off-load the reaction wheel momentum to ensure they remain within this narrow operating range, as the angular speed will continuously be evolving as the wheels are constantly gaining momentum to counteract the SRP. This off-loading is performed with the propulsion subsystem and can be done as frequently as between every target observation, in parallel to the slew to the next science target. In addition, the reaction wheels could also be accommodated on dampers to minimise all high frequency microvibrations (in the hundreds of $\mathrm{Hz}$ ) as an additional mitigation measure, as such high frequency terms cannot be accurately predicted at a Phase A level.

Similarly, the micro-vibrations from the cryo-cooler must be controlled to ensure they do not excite any structural mode of the $\mathrm{S} / \mathrm{C}$ and are sufficiently damped. With the capability to fine tune and adjust the exact operating frequency of the cooler compressors to within a few Hertz, this enables the cryo-cooler to comply with the pointing budgets allocations.

Based on this, all the fine pointing requirements have been demonstrated to be met. The only exception is the RPE which becomes marginal when also taking into the reaction wheel spikes (more accurately referred to as friction torque variations) when observing bright targets (faint targets are fine with the more relaxed RPE requirement). Such spikes have been measured on past missions and this is considered as a complex phenomenon that is due to multi-body interactions of the different balls and the distribution of lubricant within the bearing. Several mitigation measures are possible to overcome this problem, but the current baseline simply involves accepting that the science frames taken during suck spikes are lost, as this phenomenon would be sufficiently infrequent to still allow the mission to be compliant with the overall observation efficiency requirement (see section 4.2).

The propulsion system is a simple mono-propellant system (hydrazine) that is based around standard qualified off-the-shelf components (typically 1 to $20 \mathrm{~N}$ thrusters 
depending on the industrial Contractor). All thrusters are redundant, and provide control of the $\mathrm{S} / \mathrm{C}$ around the 3 rotational degrees of freedom for reaction wheels offloading and safe modes, as well as thrust for orbital correction manoeuvres.

\subsubsection{SVM electrical architecture}

The power subsystem uses standard qualified off-the-shelf components. Solar cells are accommodated on the bottom floor of the SVM which provides sufficient area to cover the needs of the mission without requiring deployable Solar arrays. The required packaging ratio of the cells leave sufficient areas for all the other units accommodated under the SVM (e.g. antennas and mechanism, Sun sensors etc.). Since the orbit is eclipse-free, batteries are only necessary during the launch phase.

The communication subsystem uses X-band only, with at least 2 Low Gain Antennas (LGA) distributed around the $\mathrm{S} / \mathrm{C}$ to provide a full $4 \pi$ steradian sky view to allow for $\mathrm{S} / \mathrm{C}$ recovery in case of a safe mode or loss of attitude. These antennas provide only a low bit rate and can therefore only be used for such recovery cases and for uplink telecommands. For science data downlink, a high bit rate is achieved with a MGA located on the bottom of the SVM. It is accommodated on a $2 \mathrm{DoF}$ mechanism to ensure that ground contact can be achieved whatever the attitude of the $\mathrm{S} / \mathrm{C}$. This enables parallel data downlink and science observations to optimise the observation efficiency budget. More importantly, it relieves any constraint on the observation plan (as the transit/eclipse observations are time critical) while allowing for fixed pre-planned ground contact times and durations in order to simplify the MOC schedule and keep the operations cost low. Any of the 3 ESA 35 m antennas (Cebreros, Malargue and New Norcia) is suitable, selection will be made closer to the launch date when the antennas' usage are better known. $14 \mathrm{~h}$ of ground contact are planned every week, split in 3 passes of $\sim 4 \mathrm{~h}$ to $6 \mathrm{~h}$. Each ground contact includes $15 \mathrm{~min}$ at its beginning and end for ranging and Doppler activities, in parallel to the full contact time for telemetry.

Memory is sized to enable continuous science operations without any loss of science data in the case of 1 missed ground contact, while the $\mathrm{S} / \mathrm{C}$ is sized to survive autonomously for at least a week in the case of 2 successive ground contacts being missed.

\subsubsection{Spacecraft budgets}

The ARIEL industrial mass budgets are presented in Table 7. Note that the PLM mass refers to the industrial alternative designs (see Fig. 9). The baseline PLM mass budget from the payload Consortium with the heavier Aluminium telescope and optical bench is indicated in section 5.2, however the SVM structural designs with the mass budgets presented here have been sized to support this heavier Consortium PLM.

For both designs, the resulting dry mass is about $\sim 1 \mathrm{t}$ (wet mass $<1.2 \mathrm{t}$ ). Note that the addition of a PLM thermal cover is currently being investigated (see discussion in section 4.1) and should result in an increase in the mass budget $\leq 50 \mathrm{~kg}$.

The ARIEL industrial power budgets are presented in Table 8 . The budgets are shown for two of the main mission modes:

- Commissioning and decontamination. This mode occurs during the first 3 months of the mission after launch, with the SVM going through its commissioning phase 
Table 7 ARIEL S/C mass budgets from the two industrial designs (in $\mathrm{kg}$ ) with their alternative PLMs

\begin{tabular}{|c|c|c|c|}
\hline & & ADS & TAS \\
\hline \multirow[t]{3}{*}{ PLM } & Telescope structure, optics and baffles & 126 & 100 \\
\hline & $\begin{array}{l}\text { Thermal shield assembly (V-grooves and struts }+ \text { others } \\
\text { (e.g. harness, mounts etc.) }\end{array}$ & 130 & 138 \\
\hline & Payload & 51 & 70 \\
\hline \multicolumn{2}{|c|}{ Total PLM } & 307 & 307 \\
\hline \multirow[t]{8}{*}{ SVM } & Warm instrument units and cryocooler & 41 & 41 \\
\hline & Structure and thermal control & 222 & 211 \\
\hline & Communications & 37 & 39 \\
\hline & CDMS & 37 & 44 \\
\hline & AOCS & 30 & 54 \\
\hline & Propulsion & 58 & 46 \\
\hline & Electrical power & 72 & 39 \\
\hline & Harness & 61 & 30 \\
\hline \multicolumn{2}{|c|}{ Total SVM } & 558 & 504 \\
\hline \multicolumn{2}{|c|}{$\mathrm{S} / \mathrm{C}$ total dry } & 866 & 812 \\
\hline \multicolumn{2}{|c|}{$20 \%$ system margin } & 173 & 162 \\
\hline \multicolumn{2}{|c|}{$\mathrm{S} / \mathrm{C}$ total dry with margin } & 1039 & 974 \\
\hline \multicolumn{2}{|c|}{ Propellant } & 107 & 138 \\
\hline \multicolumn{2}{|c|}{ Wet mass } & 1146 & 1112 \\
\hline
\end{tabular}

With the baseline Consortium PLM (and impact on the system margin), an additional $200 \mathrm{~kg}$ have to be considered

and the payload (optics and detectors at least, possibly the baffle as well) is kept warm in decontamination mode while the rest of the cryo-structure is left to passively cool down until it reaches its operational temperatures.

- Science and communications. This is the main mode used during observations in the nominal science operations phase, and allows for parallel communications with ground thanks to the antenna mechanism to optimise the observation efficiency.

Table 8 ARIEL S/C power budgets from the two industrial design for two different modes (in W)

\begin{tabular}{lllll}
\hline & Commissioning and decontamination & \multicolumn{2}{l}{ Science and communications } \\
\hline & ADS & TAS & ADS & TAS \\
Payload & 0 & 72 & 117 & 140 \\
Thermal control & 365 & 290 & 12 & 120 \\
TT\&C & 112 & 112 & 112 & 147 \\
Data handling & 61 & 53 & 103 & 73 \\
AOCS + propulsion & 38 & 151 & 38 & 138 \\
Electrical power & 46 & 20 & 45 & 20 \\
Total power without margins & 633 & 698 & 434 & 670 \\
Unit uncertainty margins & 93 & 38 & 59 & 35 \\
30\% system margin + losses & 228 & 221 & 155 & 211 \\
Total power with margins & 943 & 957 & 641 & 916 \\
\hline
\end{tabular}


Note that the exact definition of each mode varies between each contractor and therefore the budgets are not directly comparable (different units used at different times, e.g. AOCS actuators versus propulsion system, various payload units on or off during the SVM commissioning phase etc.).

Overall, these budgets indicate that the power needs of the $\mathrm{S} / \mathrm{C}$ are rather modest (under $1 \mathrm{~kW}$ ) and can be met with the bottom panel mounted solar cells described above.

\section{Spacecraft AIV and development plan}

\subsection{Model philosophy}

The spacecraft development plan is based on a standard Proto Flight Model (PFM) approach, although with some adaptations due to the specifics of the ARIEL mission: optics in cryogenic conditions, and a clear SVM / PLM separation (both physically and in terms of responsibility between ESA / industry and the payload Consortium / Member States). This implies that all final qualification tests are conducted on a S/C PFM. Such an approach carries an acceptable amount of risk and avoids having to produce and pay for two full $\mathrm{S} / \mathrm{C}$ models in parallel (one for testing / qualification and one for launch). To consider environmental tests only at final S/C FM level would further decrease the cost but would increase the risks to a level that is no longer acceptable (i.e. large cost impact if a test fails so late in the development phase and results in the need for re-design and re-qualification).

The PFM model is typically supported by additional models earlier in the development plan. The following models are baselined:

- $\quad$ an Avionics Model (AVM) to verify the electrical and electronics interfaces

- a Structural Thermal Model (STM) to de-risk the structural and thermal design with a focus on environmental testing

- a Performance Verification Model (PVM) of the PLM only under the responsibility of the payload Consortium.

Some major payload design aspects will also be de-risked earlier (starting in Phase A and continuing in Phase B1) in the program using development models where possible within the programmatic constraints.

The AVM will integrate Engineering Models (EMs) of the payload warm electronics elements (e.g. M2 mechanism electronics, Instrument and FGS Control Units, cryocooler drive electronics) that will be delivered to the $\mathrm{S} / \mathrm{C}$ prime and assembled with the SVM AVM model. This model typically enables to check all the functional and electrical (power, data and communications) interfaces between all units, to verify the functionality of the avionics including the on-board software and the AOCS closed control loop (which will require a simulator of the FGS output).

The STM allows to qualify the structure and demonstrate the thermal performance of the design. The SVM and PLM can each have a separate STM model that will be developed and tested in parallel. Mating these models into a single S/C STM is potentially not required due to the clear mechanical and thermal interface and different temperature conditions experienced by both modules. Based on heritage and fidelity of 
the structural and thermal analysis tools, the SVM STM could potentially be considered unnecessary, leaving the full qualification of the SVM structural and thermal design only to the PFM with an acceptable risk.

In addition to the AVM and STM, an additional PVM is baselined by the Consortium early in the development process, so as not to leave the full performance testing of the payload (including optics and detectors) in cryogenic conditions too late in the development process. This model will be stand-alone, i.e. will not require any other S/C or SVM units and models to mate and interface with, and as such does not require to interface with the industrial contracts and be delivered to the $\mathrm{S} / \mathrm{C}$ prime. It may include flight spares for the telescope mirrors and will use engineering models of the detector (functionally representative in terms of performance, but not flight grade) and will enable performance validation of the payload in cryogenic conditions (although some simplifications might be possible, e.g. depending on the need or not for straylight verification, the mirrors' roughness could be relaxed if not affecting the PSF quality, enabling a faster development/polishing time at a reduced cost), and more importantly validation of all payload performance software models based on correlation between test data and analysis results. If enhanced with some SVM elements, it could also be used to validate the microvibrations impact on the instrument LoS.

The final model is the PFM. It will be built from the SVM and PLM PFM models that will then be assembled together. The FM units of all payload units (telescope, instrument, cryo-cooler etc.) will be included, aligned and functionally tested in operating conditions at PLM PFM level only. Therefore, no cryogenic or optical test is needed at full S/C PFM level.

\subsection{Technology development activities}

A number of Technology Development Activities are running for ARIEL, some managed by ESA and others by the payload Consortium. They all relate to payload developments, as no need for any new technology has been identified on the SVM. Some of these are developments that are required in the baseline design, while others are only back-ups or nice-to-have for enhanced performance (these latter ones are not included in the list below). The objective of these activities is to ensure that all elements of the ARIEL design have reached a TRL $\geq 6$ by mission adoption at the end of the Phase B1 study, which is expected no earlier than Q4/2018. These are:

- Development of an Aluminium path finder telescope mirror. This activity aims at demonstrating an Aluminium primary mirror is feasible for ARIEL, considering the optical quality and cryogenic environmental requirements of the mission. In addition to the Aluminium mirror itself, demonstration of the baseline Silver coating in cryogenic conditions on this mirror is also necessary.

- Cryogenic re-focussing M2 mechanism. This activity aims at qualifying, under cryogenic conditions, a re-focussing mechanism ( $\geq 3 \mathrm{DoF})$ that will be put behind M2.

- Performance verification of a Ne JT cooler at $\leq 30 \mathrm{~K}$. This activity aims at verifying the performance of the European JT cooler (evolution from the Planck cooler) with $\mathrm{Ne}$ instead of $\mathrm{He}$ as the working fluid. In parallel, an activity dedicated to qualifying a new cooler compressor is on-going. 
- Development of European back-up low noise HgCdTe detectors and read-out electronics. Several activities exist on this topic, covering detectors with different cut-off wavelengths to cover all the channels of ARIEL (from VNIR to AIRS\#1).

\section{Science management and community access to Ariel}

ARIEL is a survey mission with the primary objective to observe a diverse sample of known, transiting exoplanets as described in chapters 2 and 3. The choice of targets shall meet the science requirements that govern the target sample, and will be made before launch. Inputs will be solicited from the wider community (e.g. through whitepapers, meetings, and other mechanisms) that will be kept informed about the status of the target list, as will the ESA Advisory Structure whose feedback will be solicited. By the time of launch it will have evolved into a description of the final nominal pre-launch ARIEL target list, however, it can and should - evolve during the mission if there are compelling scientific reasons, such as e.g. including previously unknown particularly interesting targets and actual mission in-flight performance.

ARIEL is an ESA mission with Consortium lead, it is recognized that ARIEL data and science will be of interest to a large community of 'external' exoplanetary scientists, and there is a strong wish to embrace them. The intention is to provide high quality data products in a timely manner and to have a continuous dialogue with the wider community, ultimately maximising the science that can be achieved by the mission.

The last step before commencing the nominal science operations of the mission will be the on-orbit performance verification and science demonstration phase, lasting no more than 3 months. The data from this phase will be released as soon (1-month TBC) as practical after observing, and a public workshop will be organized in connection to the data (levels 1 and 2) release.

In the nominal science operations phase the ARIEL observing strategy will be to observe the targets in three tiers as described in chapter 2 . The data will be pipeline processed to different levels of data products labelled 'raw telemetry' (level 0), 'raw spectral frame cubes' (level 1), 'target (star + planet) spectra' (level 2), and 'individual planet spectra' (level 3, respectively.

Datasets up to and including level 2 products for Tier 1 targets will be released quarterly, the objective is to achieve this 1 month after the end of each calendar quarter, e.g. 1 May for Q1 etc. Early in the mission this delay may need to be longer, if this is the case this interval will be reduced to 1 month as soon as possible as the mission progresses and a more complete understanding of the instrument characteristics, calibration needs and data processing/correction or systematics is gained.

Datasets up to and including level 2 products for Tier 2 and Tier 3 targets will be released similarly, after each semester where the required signal-to-noise (SNR) and spectral resolution for a particular target requiring multiple observations has been achieved. Early in the mission this delay may need to be longer, until the calibration needs and the knowledge needed for data processing and correction of systematics are fully understood.

Release of associated ancillary data, pipeline input files, and similar will be performed in connection with the above-mentioned releases. 
In addition, level 3 products will be produced and publicly released. These are not pipeline products but involve manual processing, and require a good understanding of ARIEL data, their instrumental signatures, and any possible peculiarities that will only be obtained once in orbit. It is in the interest of the ARIEL consortium to have level 3 data processed, published and released soon and frequently. It is foreseen to provide level 3 products at least on an annual basis, and sooner/more frequently when the knowledge to produce them is firmly in hand.

\section{Conclusions of the Ariel mission selection review}

The main conclusions of the MSR were (full details in [9]):

Technical aspects:

- The mission design was considered to be feasible and adequate to meet the mission requirements.

- At SVM level, no critical item was identified. All components have a high TRL and the overall design does not exhibit any particular complexity. The only exception is the AOCS subsystem, it will require further consolidation in the next phases, but is also expected to be feasible.

- At PLM level, the design was considered to be adequate to meet the performance requirements. A number of items however have a low TRL and will require further consolidation, but are well covered by technology development activities (see section 6.2).

- The Sun illumination of the PLM was judged to be a major risk. As a main mitigation measure, a recommendation was made to investigate the addition of a PLM thermal cover (discussed in previous sections).

- The development risk was judged to be low, but the micro-vibrations impact on the RPE budget will need to be closely monitored and might require additional characterization of the system (as opposed to verification by system analysis with characterization of units only).

Programmatic aspects:

- The main programmatic risk resides in the overall scope (a complete cryogenic PLM plus payload warm units) of the work under the responsibility of the Consortium and to be supported by the Member States.

- However the development schedule was considered consistent with a 2026 launch, and the ESA cost at completion in line with the M4 constraints.

Acknowledgments The authors wish to acknowledge the contributions by the large number of people in industry, the ARIEL consortium, and in various Directorates of ESA, whose collective effort has made this study a success.

The industrial studies for the spacecraft definition were performed by Airbus Defence \& Space and Thales Alenia Space under contract to ESA. Despite the competitive nature they have agreed to make aspects of their designs open for publication.

Finally, the enthusiasm of the exoplanetary community has always been a support. 
Open Access This article is distributed under the terms of the Creative Commons Attribution 4.0 International License (http://creativecommons.org/licenses/by/4.0/), which permits unrestricted use, distribution, and reproduction in any medium, provided you give appropriate credit to the original author(s) and the source, provide a link to the Creative Commons license, and indicate if changes were made.

\section{References}

1. Puig, et al.: The phase 0/a study of the ESA M3 mission candidate EChO. Exp. Ast. (2015)

2. Puig, et al.: ARIEL - an ESA M4 mission candidate. Proc. SPIE. 9904, (2016)

3. ARIEL Assessment Study Report, ESA/SCI (2017) 2, http://sci.esa.int/cosmic-vision/59109-arielassessment-study-report-yellow-book/

4. Tinetti et al.: "A chemical survey of exoplanets with ARIEL", Exp. Ast. (this volume)

5. Zingales et al.: "The ARIEL Mission Reference Sample", Exp. Astr. (this volume)

6. Ricker et al.: "The transiting exoplanet survey satellite", Proc. SPIE Vol. 9904, (2016)

7. Morales, et al.: Scheduling the EChO survey with known exoplanets. Exp. Astron. 40, 655 (2015)

8. Garcia-Piquet, et al.: Artificial intelligence for the EChO mission planning tool. Exp. Astron. 40, 671 (2015)

9. M4 Mission Selection Review Board report (public), ESA-SCI-F-ESTEC-RP-2017-006, https://www. cosmos.esa.int/documents/1365222/1365271/M4+MSR+Board+Report+ESA-SCI-F-ESTEC-RP-2017006+Public+Issue+1-0+Final.pdf/401667c7-a638-c2ba-3cae-85c154e4b7e9

\section{Affiliations}

Ludovic Puig $^{1}$ - Göran Pilbratt ${ }^{1}$ • Astrid Heske ${ }^{1}$ - Isabel Escudero ${ }^{1}$ - Pierre-Elie Crouzet $^{1} \cdot$ Bram de Vogeleer $^{2} \cdot$ Kate Symonds $^{2} \cdot$ Ralf Kohley $^{3} \cdot$ Pierre Drossart $^{4}$. Paul Eccleston ${ }^{5} \cdot$ Paul Hartogh ${ }^{6}$. Jeremy Leconte ${ }^{7} \cdot$ Giusi Micela $^{8}$ - Marc Ollivier $^{9,4} \cdot$ Giovanna Tinetti $^{10} \cdot$ Diego Turrini $^{11,12} \cdot$ Bart Vandenbussche $^{13}$. $^{1}$ Paulina Wolkenberg ${ }^{11,14}$

1 European Space Agency, Directorate of Science (D/SCI), ESTEC, Noordwijk, Netherlands

2 European Space Agency, Directorate of Operations (D/OPS), ESOC, Darmstadt, Germany

3 European Space Agency, Directorate of Science (D/SCI), ESAC, Madrid, Spain

4 Observatoire de Paris, Paris, France

5 Rutherford Appleton Laboratory Space, STFC, Harwell Campus, Oxfordshire, UK

6 Max-Planck-Institut für Sonnensystemforschung, Göttingen, Germany

7 CNRS / Université de Bordeaux, Bordeaux, France

8 INAF, Osservatorio Astronomico di Palermo GS Vaiana, Palermo, Italy

9 Institut d'Astrophysique Spatiale, Orsay, Paris, France

10 University College London, London, UK

11 INAF-IAPS, Rome, Italy

12 Departamento de Fisica, Universidad de Atacama, Copiapó, Chile

13 Institute of Astronomy, KU Leuven, Leuven, Belgium

14 CBK-PAN, Centrum Badań Kosmicznych Polskiej Akademii Nauk, Warsaw, Poland 